\title{
LA DIVISIÓN DEL TRABAJO DOMÉSTICO EN LAS PAREJAS ESPAÑOLAS Un análisis del uso del tiempo
}

\section{THE DIVISION OF DOMESTIC WORK AMONG SPANISH COUPLES} A time use analysis

\author{
Marta Domínguez Folgueras marta.dominguez@upf.edu \\ Universitat Pompeu Fabra. Barcelona. España
}

\begin{abstract}
Resumen
Este artículo compara la distribución del trabajo doméstico en las parejas españolas casadas y cohabitantes, contrastando las hipótesis sobre recursos relativos frente a la construcción de género y considerando si el tipo de pareja influye en la igualdad del reparto de tareas. Para examinar el grado de igualdad, se considera no sólo la contribución de cada miembro de la pareja al tiempo dedicado a las tareas, sino también quién hace qué: ciertas tareas son más restrictivas que otras y se investiga si hay diferencias de género y pareja asociadas a ellas. Los datos estudiados proceden de la Encuesta de Empleo del Tiempo (2002-2003) y se analizan mediante modelos de regresión lineal. Nuestros resultados muestran que las parejas cohabitantes tienen una división del trabajo doméstico más igualitaria, y las parejas homosexuales una división característica.
\end{abstract}

\section{Palabras Clave}

Cohabitación; Matrimonio; Parejas del mismo sexo; Roles de género; Uso del tiempo.

\begin{abstract}
This article compares the gendered allocation of household labour between married and cohabiting couples in Spain, contrasting the relative resources hypothesis and the doing gender approach, and considering that the type of partnership influences the equality of the housework division. In order to examine to what extent there is equality, we consider each partners' contribution to the total housework time, but also who does what: some tasks are more constraining than others and we investigate if there are gender and partnership differences specific to those tasks. We analyse the Spanish Time Use Survey (2003), using OLS linear regression models. Our results show that cohabiting couples are more egalitarian, and that homosexual couples have a distinctive division of labour.
\end{abstract}

\section{KEYWORDS}

Cohabitation; Gender Roles; Marriage; Same Sex Couples; Time Use. 


\section{INTRODUCCIÓN}

El trabajo reproductivo, entendido como "trabajo no remunerado que se realiza para mantener a los miembros de la familia y/o la casa" (Coltrane 2000), es clave para el funcionamiento de la sociedad. Por sus características peculiares, se ha presentado muchas veces como opuesto al trabajo productivo o remunerado, y sólo de manera reciente ha cobrado importancia su potencial impacto en la economía, como ilustra el cálculo de las Cuentas Satélite del Trabajo Doméstico (Organización de las Naciones Unidas 2000). Los dos componentes principales del trabajo doméstico son las tareas del hogar (cocina, plancha, etc.) y las labores de cuidado. Estos dos tipos de tareas se han analizado de forma conjunta, pero en este artículo nos centraremos sólo en las primeras. La idea de fondo es que los dos tipos de tareas tienen consideraciones distintas: las tareas del hogar son a menudo rutinarias, aburridas, se realizan con frecuencia por obligación y externalizarlas no tiene consideraciones negativas, mientras que en el cuidado de los hijos o dependientes existe un componente emocional elevado y es más difícil encontrar un sustituto para llevarlas a cabo.

En los países desarrollados, una de las principales características de las tareas del hogar es su asociación con el género: históricamente, se ha tratado de un trabajo mayoritariamente femenino. Con el aumento de la participación laboral de las mujeres en la última parte del siglo XX, algunos autores esperaban que se neutralizara esta especialización del trabajo por género, pero a pesar de que existen evidencias de una mayor participación masculina en el trabajo doméstico en muchos países, el reparto igualitario no es frecuente (Davis y Greenstein 2004). Estos patrones de división del trabajo según el género han llevado a la Sociología a cuestionarse las razones de este reparto y a investigar cuáles son las condiciones que propician una distribución más igualitaria. Dado que el matrimonio heterosexual ha sido la forma típica de unión hasta hace muy poco, los estudios sobre división del trabajo doméstico se centraron sobre todo en las parejas casadas. Con el aumento de los hogares alternativos, la unidad de análisis se ha ido ampliando para incluir a los cohabitantes y las parejas homosexuales. Y los resultados, para varios países, han mostrado que las parejas no casadas tienen una división del trabajo doméstico más igualitaria que los casados (Batalova y Cohen 2002), y que algo parecido ocurre con las parejas del mismo sexo (Kurdek 2001).

Gran parte de la literatura sobre las diferencias en la distribución del trabajo doméstico que tiene en cuenta la estructura familiar utiliza datos de Norteamérica o los países escandinavos, pero no disponemos de estudios similares a nivel nacional para el caso español. Se trata de un caso interesante por la rapidez de los cambios producidos en cuanto a comportamiento y valores: mientras que los valores se han hecho más tolerantes, este cambio no se ha reflejado tanto en el comportamiento familiar; la esfera pública se ha movido hacia valores de género más igualitarios, y esto ha producido cambios en la esfera privada (Meil 1997), cuyo alcance está aún por determinar. En esta investigación consideramos el reparto del trabajo doméstico en España, teniendo en cuenta las 
teorías existentes y los resultados de estudios previos, a los que añadimos dos aportaciones: tenemos en cuenta el tipo de pareja como variable explicativa y consideramos que a la hora de estudiar la igualdad no se trata sólo de calcular el tiempo total dedicado a las tareas del hogar, sino también de ver las diferencias que se producen según el tipo de tarea que se lleva a cabo.

La estructura del artículo es la siguiente: en primer lugar se revisa la literatura sobre la división del trabajo doméstico, así como sobre las diferencias entre tipos de parejas y su relación con las tareas del hogar. A continuación se describen los resultados previos sobre el caso español y las hipótesis a estudiar. En la sección siguiente, se presenta el análisis de datos, que contiene elementos descriptivos y un análisis de regresión utilizando datos de uso del tiempo. Los resultados se discuten en la última sección del artículo.

\section{MARCO TEÓRICO}

\section{Género y trabajo doméstico}

En los años 90 el estudio del trabajo doméstico se convirtió en un área importante de las ciencias sociales, y pronto se estableció su relación con otros fenómenos sociales: las relaciones familiares, el mercado laboral, los valores y determinados acontecimientos del ciclo vital (Coltrane 2000). Este tipo de trabajo presentaba una fuerte asimetría de género, de acuerdo con el modelo del male breadwinner, según la cual el hombre trabajaba fuera de casa y la mujer se ocupaba del hogar y los hijos. Este tipo de familia fue mayoritaria durante gran parte del siglo XX, y varias teorías sociológicas proporcionaron posibles razones para la permanencia de dichos patrones de especialización. En este artículo nos centraremos en la perspectiva micro, que intenta explicar las decisiones de la familia y la interacción entre los miembros sobre el reparto de tareas dentro del hogar. Aunque presentan variaciones notables, podemos agrupar estas teorías en dos grupos: las centradas en los recursos relativos y las que apuntan a los procesos de socialización. Se trata de teorías que parten de supuestos diferentes y que, por lo tanto, supondrían explicaciones teóricamente excluyentes.

Dentro de las teorías sobre los recursos relativos, el primer representante que suele citarse es Becker (1987) y la nueva economía del hogar, que interpretó la especialización de las mujeres en las tareas del hogar como un resultado del capital humano y las características del mercado laboral. Puesto que las mujeres tenían un nivel educativo menor, su participación en el mercado de trabajo llevaba asociados unos ingresos esperados más bajos. En cambio, los hombres tenían ventajas relativas en el mercado laboral, por lo que era razonable que el hogar, como unidad, se decantara hacia un modelo en el que las mujeres se especializaran en mayor o menor grado en el trabajo no remunerado y en la crianza de los hijos. La permanencia de este modelo de familia depende de otros 
factores económicos, por lo que un cambio en los mercados laborales o incrementos en la formación de las mujeres podría provocar cambios en los modelos de especialización, y por eso se considera que la teoría de Becker es neutral en lo que se refiere al género.

El retrato beckeriano de la división del trabajo en la familia se refinó a través del concepto de negociación o bargaining (Lundberg y Pollak 1996) según este enfoque, las decisiones en el hogar no se toman como si este fuera una unidad con una función de utilidad única, sino que cada miembro tiene sus preferencias y debe negociar para llegar a un equilibrio. El poder de negociación de cada individuo depende de su potencial aportación al hogar, que suele medirse en términos de ingresos. Con la incorporación de las mujeres a los niveles educativos más elevados y al mercado laboral a tiempo completo, su poder de negociación dentro del hogar aumenta, lo que implicaría una disminución de su tiempo dedicado al trabajo doméstico a favor de un reparto más igualitario. Este enfoque de la negociación supone un avance respecto al primero porque, al considerar que los miembros de la familia pueden tener preferencias personales y funciones de utilidad propias, se acerca más a la complejidad que se da en las relaciones humanas. Sin embargo, dentro de este enfoque los factores que influyen en la capacidad de negociación son básicamente los mismos recursos planteados por Becker: educación e ingresos.

No todos los recursos que influyen en el tiempo dedicado al trabajo doméstico se relacionan con los ingresos o la capacidad de tener éxito en el mercado laboral; por ejemplo, un factor más inmediato y que claramente influye es el tiempo disponible. Aunque aquí lo consideramos como un recurso más, la disponibilidad temporal se suele considerar como una teoría separada, según la cual el miembro del hogar que trabaja más horas fuera de casa dispondrá también de menos tiempo para realizar las tareas y por tanto dedicará menos tiempo a estas (Presser 1994). Otro recurso que se ha considerado relevante para explicar la división del trabajo doméstico es el capital social, que se ha relacionado con la posibilidad de modificar el reparto del trabajo productivo y reproductivo si el capital humano de uno de los miembros de la pareja se transfiere al otro (Balcells 2009).

Así pues, de acuerdo con las diversas teorías centradas en los recursos relativos, el factor que mejor puede predecir la dedicación a las tareas del hogar sería la situación laboral: ya sea por los ingresos que reporta, que aumentan el poder negociador del que los percibe, o bien por la reducida disponibilidad de tiempo que implica. Cabría esperar que, a mayor dedicación al trabajo remunerado, menor implicación en el doméstico. En este sentido estas teorías han encontrado apoyo empírico: las mujeres que trabajan a tiempo completo hacen un porcentaje menor de tareas que las mujeres que no trabajan (Gershuny 2000), y cuando ambos miembros de la pareja son laboralmente activos, tienen un reparto más justo (2000), con diferencias que dependen del tipo de participación en el mercado y del tiempo disponible (Presser 1994). Las mujeres con ingresos más elevados también tienen una división más justa, aunque lo importante es el peso relativo de sus ingresos dentro del hogar. En lo que respecta a los hombres, los resultados no son tan claros: parece que existe evidencia a favor de la disponibilidad de tiempo, 
porque los que trabajan menos horas fuera de casa realizan más tareas (Bianchi et al. 2000). Sin embargo, algunos autores han señalado que la división del trabajo doméstico no se ha hecho más justa, ya que los hombres no han aumentado su participación de forma significativa: el descenso en la proporción de trabajo doméstico que llevan a cabo las mujeres se debe a que ellas hacen menos, y no a que los hombres hagan más; en algunos casos, las mujeres laboralmente activas aumentan su contribución al trabajo doméstico cuando su pareja se queda sin trabajo (Brines 1994), y este es un hecho difícil de explicar desde la teoría de los recursos relativos. Estas teorías tampoco pueden explicar la persistencia de la especialización de género en las parejas con dobles ingresos. Un problema adicional es que las teorías de los recursos relativos suelen asumir que las mujeres con niveles educativos elevados prefieren repartos igualitarios en el hogar y trabajar fuera de casa, y por lo tanto interpretan como una anomalía o un fracaso de la negociación la realidad contraria, pero no podemos olvidar que incluso las mujeres (y los hombres) que han hecho una inversión importante en capital humano pueden tener preferencias variadas en cuanto a la conciliación de trabajo y familia, y orientarse preferiblemente hacia uno de los dos caminos, o bien estar dispuestas a adaptarse a las circunstancias, como señala Hakim (2000).

Una explicación alternativa a los recursos relativos se centra en los procesos de socialización y en el establecimiento de roles de género. Según las teorías de la socialización, la mayor contribución de las mujeres al trabajo doméstico no se debe a las características del mercado laboral y a la aplicación de criterios racionales dentro del hogar, sino a su propia conciencia de género y a las normas sociales existentes. La identidad de género comienza a fijarse desde la infancia: las mujeres aprenden que se espera de ellas precisamente un perfil especializado en las tareas domésticas, y se trata de un proceso complejo, reforzado por diversas instituciones, en niveles diversos. En concreto, basándose también en principios de socialización, las teorías sobre la construcción de género, doing gender, sostienen que la identidad de género se construye de forma dinámica, a través de la interacción diaria (West y Fenstermaker 1993). Puesto que las tareas domésticas están asociadas a lo femenino, las mujeres continúan realizando más trabajo doméstico porque esto forma parte de la construcción de su identidad de género, de su feminidad.

Esta teoría proporcionaría argumentos para explicar la supervivencia de la especialización de las mujeres en el trabajo doméstico a pesar del aumento del poder de negociación de estas, que constituía una evidencia conflictiva para las teorías de recursos relativos. Sin embargo, las implicaciones empíricas de las teorías del doing gender son más difíciles de operacionalizar, ya que es difícil detectar cambios en los roles esperados de cada sexo, y más aún en las percepciones individuales de estos. Un posible indicador son los valores ideológicos y de género; cuando existe información sobre ellos, muestran una relación coherente con el reparto del trabajo doméstico: las parejas con valores más igualitarios tienen un reparto más igualitario que las demás (Lennon y Rosenfield 1994; Lück y Hofäker 2003; Meil 2005). Otro factor que se asocia al cambio de valores o a la ruptura con lo establecido es la educación, y se trata de una variable con 
un importante impacto sobre la división de tareas: las mujeres con niveles educativos más altos realizan un porcentaje menor de las tareas del hogar, mientras que el efecto es el contrario para los hombres: a mayor nivel educativo, más proporción de las tareas realizan (Anxo 2002; Bianchi et al. 2000; Gershuny 2000; Perkins y de Meris 1996; Pittman 1995; entre otros). A pesar de ello, hay que ser cautelosos a la hora de interpretar los resultados referidos a la educación, ya que esta puede ser un indicador de los valores, pero también es una medida asociada a clase social, capital humano y recursos relativos.

Así pues, existe evidencia a favor y en contra de ambos tipos de teorías en estudios que se han ocupado de varios países. Concretamente, para el caso español los análisis previos han detectado que las mujeres se enfrentan a una doble carga laboral, dentro y fuera de casa (González 2001) y que se da una influencia importante de la participación en el mercado laboral, los valores y el nivel educativo (Meil 1989; Balcells 2009), lo cual se ha interpretado como evidencia a favor de la teoría de los recursos relativos. También se ha mostrado que las parejas no casadas tienen una división del trabajo más igualitaria, como ocurre en otros países (Meil 2003; Domínguez y Castro 2008). Todo esto nos indica que ambos tipos de teorías no son necesariamente incompatibles sino complementarias. La división del trabajo doméstico puede verse influida por factores sociales como los mercados laborales y la capacidad de negociación de las parejas, pero esto no implica que haya que descartar el peso de la socialización y los roles de género. Junto con estas variables, hay más factores que influyen en la división del trabajo doméstico, como diversos acontecimientos del ciclo vital, aunque los cambios que implican solo pueden observarse si se dispone de datos longitudinales. Especialmente importante es el nacimiento de un hijo, que parece reforzar la división del trabajo hacia roles más tradicionales incluso en parejas con actitudes más igualitarias (Gjerdingen 2005; Dribe 2009). Abandonar el mercado laboral de forma permanente también aumenta la dedicación al trabajo doméstico (Pittman 1995), pero esto puede deberse principalmente a que aumenta la disponibilidad temporal.

\section{El trabajo doméstico}

Hasta este punto, hemos considerado el trabajo doméstico como algo unitario, sin embargo no está claro que esto sea lo más acertado. El trabajo doméstico implica muchas tareas con características diferenciadas: cocinar, planchar o hacer la compra no son actividades igualmente satisfactorias 0 aburridas. Algunas tareas son más placenteras, algunas necesitan más tiempo que otras, y además, algunas deben llevarse a cabo cada día, con frecuencia en un horario determinado, y son difíciles de posponer. Tareas como limpiar la casa o tender la ropa suelen caracterizarse como tareas "centrales", "rutinarias" o "repetitivas", como contrapuestas a tareas como hacer pequeñas reparaciones, cuidar las plantas o comprar objetos necesarios para la casa, que son tareas realizadas de manera ocasional, más cercanas al ocio y más flexibles y fáciles de posponer. La división de tareas domésticas también sigue un eje de género en este sentido, ya que se ha observado que las mujeres se concentran más en las tareas rutinarias y los hombres en 
las ocasionales, de forma que algunos autores incluso denominan a las primeras "tareas femeninas" y a las segundas "tareas masculinas" (Thompson y Walker 1989; Bianchi et al. 2000). Así pues, para saber si la división de tareas es igualitaria, nos interesa saber quién se encarga de cada tarea en mayor medida, y no sólo cuánto tiempo se dedica en total al trabajo doméstico dentro de la pareja.

El cuidado de los hijos se suele incluir como una tarea doméstica más en muchos estudios. Sin embargo, no está claro que se trate de una tarea del hogar comparable a las que acabamos de presentar. El cuidado de los hijos conlleva un componente emocional, es difícil de externalizar y es una actividad valorada positivamente por los padres, es decir, que tiene un componente de disfrute que no está presente en las tareas rutinarias como por ejemplo hacer la limpieza. Además, la literatura reciente ha mostrado que los padres no intentan repartir el cuidado de los hijos, sino realizar actividades con los hijos de forma conjunta (Hallberg 2003; Sayer 2005), lo cual apunta a que consideran el cuidado de los niños como algo más cercano al ocio. Por estas razones consideramos que las decisiones sobre cómo repartir el cuidado de los hijos pueden seguir patrones diferentes y no las incluiremos en el estudio.

\section{Trabajo doméstico y tipo de pareja}

Con la difusión de nuevos modelos de familia, las parejas no casadas se convirtieron en un escenario alternativo para estudiar la división del trabajo doméstico. De hecho, la literatura sugiere que las parejas no casadas reparten el trabajo doméstico de forma más igualitaria que las casadas (Batalova y Cohen 2002; Baxter 2005; Shelton y John 1993; South y Spitze 1994). Las explicaciones para esta regularidad empírica son diversas: en primer lugar, las parejas que cohabitan tienen unos valores de género más igualitarios que las que prefieren casarse (Clarkberg et al. 1995; Castro y Domínguez 2008) aunque algunos estudios longitudinales muestran un cambio en la división de las tareas hacia roles más tradicionales cuando las parejas cohabitantes se casan (Gupta 1999). Además, la duración de este tipo de uniones (más breve que los matrimonios) podría reducir el interés de las mujeres en especializarse en el trabajo reproductivo (Clarkberg 1999). Esta última justificación se relaciona con la naturaleza de las uniones no casadas y con el significado que se les atribuye. En la mayoría de países europeos, salvo en los escandinavos, sólo un pequeño porcentaje de las uniones no casadas continúa como tales de forma permanente, la mayoría termina en ruptura o en matrimonio. Si uno de los significados que se le atribuyen a este tipo de uniones (Heuveline y Timberlake 2004) es el de un nuevo elemento del proceso de selección de parejas, y una parte de ese proceso puede estar relacionada con la disposición de la pareja a compartir las tareas, la voluntad de compartir puede ser una característica muy valorada en parejas potenciales, sobre todo por parte de mujeres comprometidas con su trabajo. Esta característica se valorará especialmente en sociedades que mantengan un grado de desigualdad relativamente elevado en la esfera privada (Ono 2003), como parece ocurrir en el caso español (González 2001). El reparto del trabajo doméstico es una variable importante 
en nuestro país a la hora de determinar la estabilidad de la pareja (Meil 2005), lo que indica que puede ser un factor de selección interesante. En resumen, existe evidencia sobre los valores de género más igualitarios entre las parejas de hecho, pero la causa de esta diferencia con los casados no está clara, ya que podría deberse a un efecto de selección -parejas con valores más igualitarios prefieren no casarse-, o a la propia naturaleza de la relación - si funciona como un proceso de selección en el que los hombres prefieren mostrarse más igualitarios.

Además de la difusión de las parejas no casadas, una nueva forma de familia que ha adquirido relevancia social la constituyen las parejas homosexuales, casadas 0 no. Este tipo de parejas constituyen un caso de estudio privilegiado para el reparto de tareas porque nos permitirían distinguir el peso de factores relacionados con los recursos frente a la socialización. En estas parejas, ambos miembros son del mismo género, por lo tanto han sido socializados en valores similares y es mucho más probable que tengan ideas parecidas sobre la división del trabajo doméstico. En principio, los dos miembros han experimentado una construcción parecida, cuya preferencia acerca de las tareas se diferenciarán sólo por factores que son ajenos a la inter-construcción de género (Kurdek 2001). Ahora bien, es posible que el proceso de socialización de género en el caso de los niños homosexuales sea diferente: podría producirse un rechazo de los roles aprendidos, y las propias familias podrían mostrar actitudes diferentes hacia los jóvenes debido a su orientación sexual (Solomon et al. 2005). Estas posibles divergencias en la socialización de género tendrán como resultado una mayor heterogeneidad de roles e identidades de género entre las parejas del mismo sexo, frente a una identidad más uniforme en el caso de las parejas heterosexuales. Debido a la todavía reducida presencia de las parejas del mismo sexo en las encuestas, la bibliografía sobre la división del trabajo doméstico entre estas parejas es menos extensa, pero hasta el momento los estudios muestran una división del trabajo doméstico mucho más igualitaria (Kurdek 2001; 2007; Solomon et al. 2005; Sullivan 1996) y más dependiente del poder de negociación económico (Sullivan 1996).

Así pues, a la hora de estudiar la igualdad del reparto de las tareas domésticas en las familias españolas, debemos tomar en consideración que se ha encontrado evidencia empírica a favor de las hipótesis de los recursos relativos pero también de las teorías sobre la construcción de género, que el reparto puede ser desigual según el tipo de tarea y que tenemos razones para esperar un reparto diferente según el tipo de pareja. Teniendo esto en cuenta, las principales hipótesis que intentaremos comprobar en el estudio empírico son las siguientes:

(H1) Los recursos relativos son importantes para determinar la proporción de trabajo del hogar: trabajar a tiempo completo, tener un nivel educativo alto, y ganar más dinero que la pareja reducirán la proporción de tareas del hogar realizadas, independientemente del género.

(H2) Si la construcción de género es relevante, es muy posible que observemos efectos diferentes, en magnitud y dirección, en las variables consideradas entre hombres y mujeres. 
(H3) Las mujeres hacen la mayoría del trabajo doméstico en ambos tipos de pareja, pero las parejas no casadas tienen un reparto más justo. Es decir, los hombres en las uniones no casadas realizarán una mayor proporción de tareas, y en particular, harán más tareas rutinarias que los casados (controlando otros factores).

$(\mathrm{H} 4)$ Las parejas homosexuales tendrán una división de las tareas igualitaria y las diferencias entre los miembros de la pareja dependerán de sus recursos.

\section{Datos y metodología}

La medición de las tareas del hogar resulta problemática (Lee y Waite 2005; Twiggs et al. 1999); muchas veces se recoge información de manera indirecta, a través de preguntas sobre el tiempo aproximado dedicado a esas tareas, o sobre quién es el principal responsable de dichas actividades. La información disponible para el caso español hasta ahora tenía ese formato, pero la Encuesta de Uso del Tiempo nos proporciona los datos más precisos hasta el momento sobre nuestra variable dependiente: el tiempo total dedicado a las tareas del hogar. La encuesta fue realizada por el Instituto Nacional de Estadística (INE) en 2002-2003, dentro del proyecto internacional de Encuestas Armonizadas de Uso del Tiempo'. La encuesta española se basa en una muestra de 24.000 hogares, aunque en nuestro análisis incluimos sólo parejas de entre 18 y 65 años, por lo que la submuestra se reduce a 19.182 individuos. Además de proporcionar información sobre variables socioeconómicas (estatus laboral, nivel educativo e ingresos) y características de los hogares (relaciones entre todos los miembros del hogar y equipamientos de este), cada miembro del hogar debe rellenar un diario de actividades. Estos diarios dividen el día en periodos de 10 minutos, para cada uno de los cuales el encuestado debe especificar qué actividad llevó a cabo durante ese periodo. En cada uno de esos intervalos se recoge información sobre actividades paralelas 0 secundarias - por ejemplo si el individuo estaba cocinando y escuchando la radio a la vez-, en qué lugar se econtraba el individuo en ese momento, y si había otro miembro del hogar presente.

Mientras que la información disponible hasta el momento sobre el reparto de las tareas del hogar se basaba en preguntas acerca de quién es el principal responsable de una tarea (Balcells 2009), los datos de uso del tiempo nos proporcionan una aproximación al tiempo real dedicado a las tareas y nos permiten ver, en un día concreto cuánto tiempo se dedica a cada una de ellas, con lo que no dependemos de percepciones subjetivas a la hora de interpretar el reparto. Sin embargo, este tipo de encuestas no están exentas de problemas. Los tipos de actividades se codifican según un catálogo, que pese a su amplitud, nunca refleja todas las actividades que

\footnotetext{
${ }^{1}$ http://www.timeuse.org/mtus/
} 
el investigador desearía ver recogidas. Por otra parte, no tenemos la seguridad de que los individuos respondan fielmente al cuestionario, y en algunas actividades cuya duración puede ser inferior a los diez minutos puede ser difícil para los encuestados especificar las respuestas. Sin embargo, estos problemas afectan a cualquier pregunta de encuesta acerca del uso de tiempo o de la realización de tareas domésticas que no se base en la observación directa del investigador. En el caso español, solo disponemos de datos de un día, a diferencia de otros países que recogen datos para dos o tres días, lo que permite saber, por ejemplo, si las tareas se compensan en días sucesivos. Una desventaja adicional de la encuesta española es que no incluye información acerca de los valores de género, por lo que no podemos considerar directamente si las diferencias observadas se deben a esto.

Las diferencias en el tiempo total que dedican a las tareas del hogar hombres y mujeres son interesantes de por sí, pero en este caso nos interesa el reparto entre los miembros de la pareja, y por eso recodificamos la variable dependiente, que es la contribución de cada individuo al total de tiempo que destina a su hogar: calculamos primero el total de cada hogar y después la proporción que realiza cada uno de sus miembros. Por ejemplo, tomemos dos hogares formados por parejas heterosexuales en los que ambos trabajan fuera de casa; en el primero la mujer dedica 300 minutos al trabajo doméstico y en el segundo 100. Si en el primer hogar el hombre dedica 100 minutos, esto significa un reparto muy desigual ( 75 por ciento frente al 25 por ciento), puesto que la mujer hace tres veces más que él, mientras que en el segundo hogar esa misma dedicación total supondría un reparto igualitario perfecto (50 por ciento cada uno). Por esta razón, en lugar del tiempo total, lo que tenemos en cuenta es el porcentaje del total que hace cada uno de los miembros de la pareja, controlando también si reciben alguna ayuda. No obstante, quizá es más fácil que el reparto sea igualitario cuando hay menos tareas que repartir, y para comprobar esto en el análisis controlamos la cantidad total de tiempo que dedica el hogar a cada tarea.

Esperamos encontrar diferencias específicas según el tipo de tarea, por lo que tomamos en consideración cuatro tipos principales de actividades, además del total. La actividad más rutinaria es la "limpieza", que incluye la limpieza del hogar, los platos, colada y plancha, mientras que "cocina" incluye la preparación de las comidas, poner y retirar la mesa. Una actividad menos rutinaria es la que denominamos "compras", que incluye la compra de alimentos, pero también de cualquier objeto necesario para la casa, así como gestiones domésticas (pago de recibos, por ejemplo). Finalmente, la última categoría, que llamamos "ocasionales" incluye las actividades consideradas masculinas en la literatura, más cercanas al ocio, como las pequeñas reparaciones, el cuidado del jardín y de las mascotas. Utilizamos para esto solo las actividades que el encuestado ha anotado como primarias. Las tareas del hogar como actividades secundarias representan sólo un dos por ciento del total de tiempo dedicado a tareas, y a menudo la actividad primaria 
anotada es otra tarea del hogar ${ }^{2}$. No incluimos el cuidado de los niños como variable dependiente porque, como hemos mencionado en la primera parte de este artículo, no es puramente una tarea del hogar; no obstante, es muy probable que el tiempo dedicado al cuidado de los hijos forme parte de las negociaciones de las parejas acerca de la distribución del trabajo doméstico, ya que los miembros de la pareja podrían intercambiar tiempo de cuidados por tiempo dedicado a las tareas. Con la idea de incluir en el análisis la proporción del cuidado de los hijos como variable de control, estudiamos su correlación con la variable dependiente, y encontramos que su nivel de correlación es muy alto (superior a 0,6), es decir, que las personas que hacen mayor proporción de tareas del hogar también hacen mayor proporción de cuidado. Dada esta correlación, parece que no existe un efecto sustitución de un tipo de tareas por otras, y por eso no incluimos este control en el análisis; aunque sí introducimos controles relativos a la presencia de niños, su número y edad.

Los análisis para las parejas heterosexuales y del mismo sexo son diferentes. En el caso de las parejas heterosexuales, la variable dependiente es el porcentaje de las tareas realizado por cada uno de los miembros y nuestra principal variable independiente es el tipo de pareja: matrimonio o cohabitación. Otras variables de interés son el nivel educativo (primario, secundario o universitario), la actividad en el mercado de trabajo (a tiempo completo, parcial o sin actividad), la ayuda doméstica recibida por el hogar, y si el cuestionario se completó en fin de semana, momento en el que aumenta el tiempo dedicado para todos y para determinadas tareas. También introducimos controles por edad (edad y edad al cuadrado), la presencia de niños menores de 14 años y su número, así como las características de la pareja (en términos de nivel educativo, participación laboral y diferencia de ingresos si ambos trabajan). Hombres y mujeres son analizados por separado para poder establecer las diferencias de género.

En cuanto a las parejas del mismo sexo, podemos identificarlas en la encuesta aunque no haya una pregunta directa, ya que sabemos qué personas forman una pareja y además conocemos el sexo de todas las personas residentes, los componentes en el hogar. Los resultados que obtengamos deben tomarse con precaución puesto que están representados solo por 148 casos. Para poder incluir todos los casos en el análisis, en este caso tomamos como variable dependiente la cantidad total de minutos dedicados a las tareas y no la proporción ${ }^{3}$. Para aumentar los grados de libertad no incluimos en el análisis la actividad laboral ni el nivel educativo de la pareja. En este caso no realizamos análisis separados por sexo pero incluimos la variable como control.

Un examen preliminar de los datos nos indica que existe un reparto desigual entre géneros y asimetrías entre los tipos de pareja, pero para poder controlar los factores asociados con la división del trabajo doméstico utilizamos un análisis de regresión. En

\footnotetext{
${ }^{2}$ No obstante, se analizaron los datos teniendo en cuenta este tipo de actividades como un grupo separado, pero no se obtuvieron resultados significativos.

${ }^{3}$ Si estudiáramos la proporción solo podriamos incluir a uno de los miembros de la pareja en el análisis.
} 
la literatura sobre uso del tiempo existe un debate acerca de la conveniencia de utilizar regresión lineal frente a modelos tobit. Los últimos permiten controlar posibles efectos de selección en los casos en los que hay muchas observaciones con valor 0 , es decir, que no dedican ningún tiempo a la actividad a estudiar. En nuestro caso hemos llevado a cabo los dos tipos de análisis y los resultados han sido similares. Puesto que no tenemos razones teóricas para esperar un sesgo en las observaciones con valor 0 , y dado que la interpretación de los coeficientes de una regresión lineal es más sencilla, estos serán los análisis presentados en el artículo. Por razones de espacio no se incluyen los resultados de los modelos tobit, pero estos resultados pueden solicitarse al autor.

\section{Resultados}

Según las encuestas de opinión, los españoles tienen una actitud bastante abierta hacia las nuevas formas de familia y los roles de género igualitarios. El 65 por ciento de los entrevistados por el Centro de Investigaciones Sociológicas (CIS) en 2008 elegían como forma de familia ideal aquella en la que los dos miembros de la pareja trabajaban fuera de casa con dedicación parecida y compartían las tareas de forma igualitaria (Estudio 2766); en 2004 el 57 por ciento aprobaba el matrimonio homosexual y el 77 consideraba que los cohabitantes debían disfrutar de las mismas ventajas legales que los casados (CIS, Estudio 2578); en 2008 el 65 por ciento encontraba aceptables las relaciones homosexuales (Estudio 2752). Estos puntos de vista son más fuertes entre las cohortes más jóvenes, pero a pesar de ello, las familias "alternativas" todavía son una minoría en España: en 2006 (Estudio 2369), solo un 12 de la población menor de 45 años vivía en pareja sin casarse, y según el Censo 2001 el porcentaje de parejas homosexuales estaría entre el 1 y el 3 por ciento. Las parejas no casadas constituyen un grupo selectivo de la población, con un perfil socioeconómico distinto de la media, o al menos así era en el Censo 2001: se trataba de parejas más jóvenes, con mayor nivel educativo, menos hijos y con una actividad laboral femenina más alta que la de las mujeres casadas (Castro y Domínguez 2008), perfil que encontramos también en la muestra de la encuesta analizada en el presente artículo (ver Tabla del Anexo).

En cuanto a los roles de género, actualmente las mujeres españolas han alcanzado una tasa de participación laboral elevada (un 55 por ciento en 2008 según Eurostat), a pesar de mantener una tasa de paro superior a la de los hombres (13 por ciento). La Encuesta de Uso del Tiempo nos indica que, aunque haya habido importantes avances hacia la igualdad en la esfera pública, dentro de los hogares las mujeres siguen dedicando más tiempo que los hombres a las tareas domésticas: 84 por ciento de las mujeres cocinaban a diario, el 71 por ciento limpiaba; mientras que para los hombres estos porcentajes son inferiores: solo el 44 por ciento cocinaba regularmente y el 28 por ciento llevaba a cabo tareas relacionadas con la limpieza; otras tareas, como encargarse del jardín o de lavar el coche, son más masculinas. En cuanto al tiempo total invertido, 
la media de las mujeres dedica unas 5 horas al día a tareas del hogar; las que reciben ayuda externa, una hora menos.

La tabla 1 resume la dedicación a diversas tareas, por sexo y tipo de pareja: matrimonio heterosexual, cohabitación heterosexual y pareja del mismo sexo. Las tres primeras filas muestran el número de minutos invertidos en cada actividad, mientras que las dos últimas líneas muestran el porcentaje total realizado por cada sexo, sobre el total del hogar. Nótese que en este último caso no tiene sentido calcular el porcentaje realizado por cada miembro de las parejas del mismo sexo ya que por composición este siempre es un 50 por ciento. Si observamos el total de minutos, es evidente que las mujeres dedican el triple de tiempo a las tareas del hogar que los hombres, y que se da una diferencia entre casados y cohabitantes, aunque pequeña. Para las distintas actividades, los hombres en una unión no casada dedican más tiempo a todas ellas que los hombres casados; la única diferencia reside en la categoría de "ocasionales", en la que los cohabitantes invierten menos tiempo que los casados. Las mujeres no casadas también dedican menos tiempo que las casadas a todas las tareas, pero dedican un poco más a compras y gestiones y a las ocasionales. En cuanto a las parejas del mismo sexo, el número de minutos que pasan realizando tareas del hogar está más igualado y en torno a los 200 minutos. Las mujeres dedican algo más que los hombres, especialmente en las tareas de limpieza, pero el tiempo invertido en las demás categorías es similar; ligeramente superior para las mujeres en el caso de las actividades ocasionales.

Tabla 1.

Tiempo total en minutos y contribución en porcentaje dedicado a las tareas del hogar, por sexo y tipo de pareja.

\begin{tabular}{lcccccccccc}
\hline & \multicolumn{2}{c}{ Total } & \multicolumn{2}{c}{ Limpieza } & \multicolumn{2}{c}{ Cocina } & \multicolumn{2}{c}{ Compras } & \multicolumn{2}{c}{ Ocasionales } \\
\cline { 2 - 11 } & \multicolumn{1}{c}{ Hombres } & Mujeres & Hombres & Mujeres & Hombres & Mujeres & Hombres & Mujeres & Hombres & Mujeres \\
\hline Casados & 80 & 279 & 14 & 108 & 22 & 128 & 18 & 34 & 26 & 9 \\
Cohabitantes & 89 & 263 & 16 & 103 & 31 & 116 & 20 & 37 & 22 & 10 \\
Mismo sexo & 187 & 209 & 94 & 124 & 76 & 72 & 28 & 31 & 18 & 26 \\
\hline Porcentaje del hogar (\%) & & & & & & & & & \\
\hline Casados & 21 & 79 & 12 & 88 & 15 & 85 & 31 & 69 & 69 & 31 \\
Cohabitantes & 31 & 69 & 21 & 79 & 28 & 72 & 37 & 63 & 60 & 40 \\
\hline
\end{tabular}

Fuente: Elaboración propia a partir de datos de la Encuesta de Empleo del Tiempo 2002-2003 
Si nos fijamos en las dos últimas filas, que reflejan la contribución relativa, las diferencias entre tipos de pareja son significativas: los hombres que cohabitan hacen una tercera parte de las tareas, frente a los casados, que hacen un 21 por ciento. En particular, los no casados hacen una proporción mayor de limpieza y cocina, que son las tareas consideradas más rutinarias 0 aburridas en la literatura. A su vez, realizan una proporción menor de las ocasionales, y estos porcentajes nos indican que, en términos agregados, se da una división del trabajo doméstico más igualitaria entre las parejas no casadas, ya que los hombres son responsables de una mayor proporción de tareas, y además de tareas consideradas "femeninas". Ahora bien, las diferencias que observamos a nivel agregado pueden deberse a ciertos factores asociados con el tipo de pareja, por ejemplo, al hecho de que las mujeres no casadas sean más jóvenes y tengan valores menos conservadores, 0 a la menor participación laboral de las casadas, que aumenta su disponibilidad de tiempo.

Para controlar estos factores analizamos los datos con regresiones lineales en las que, en el caso de parejas heterosexuales, nuestra variable dependiente es la proporción de las tareas que lleva a cabo cada individuo. En este apartado describiremos brevemente los resultados, que se comentarán en la sección siguiente. La tabla 2 presenta los coeficientes obtenidos para los hombres y la tabla 3 para las mujeres. La primera columna muestra los efectos de las variables sobre el porcentaje de tareas total, las columnas siguientes para actividades específicas. A la hora de interpretar los resultados, es importante recordar que la variable dependiente es la contribución individual en porcentaje sobre el total, por lo tanto los coeficientes no se traduce directamente en cantidades de tiempo, sino en incrementos o disminuciones del porcentaje realizado. En la tabla 2, la primera columna nos muestra que todas las variables analizadas tienen un efecto significativo sobre la proporción de tareas realizadas. Vivir en una pareja no casada, tener un nivel educativo más elevado, trabajar menos horas - a tiempo parcial 0 no trabajar-, tener una pareja con alto nivel educativo, y recibir ayuda doméstica aumentan la proporción de tareas realizadas. De todas estas variables, el efecto más fuerte es el de la actividad laboral a tiempo parcial, que aumenta la colaboración en un 11 por ciento y el del nivel educativo universitario, que la aumenta en un 6,24 por ciento. La contribución del hombre aumenta los fines de semana, pero en un porcentaje que no llega al 6 por ciento. En cambio, si la mujer trabaja menos horas, los hombres reducen su colaboración, y lo mismo ocurre si él gana lo mismo o más que ella (tomando como referencia la situación más común, que los ingresos del hombre sean más altos). Si examinamos los modelos para tareas específicas, el efecto de las variables de control es similar al descrito para la primera columna, pero los efectos son más fuertes en el caso de las tareas menos rutinarias. El tiempo total que dedica el hogar a las tareas domésticas tiene un efecto significativo y positivo sobre la proporción realizada por los hombres, pero es un efecto que no alcanza el 0,5 por ciento. 
Tabla 2.

Resultados del análisis de regresión sobre contribución a las tareas (hombres)

\begin{tabular}{|c|c|c|c|c|c|}
\hline & Tiempo total tareas & Limpieza & Cocina & Compras & Ocasionales \\
\hline Edad & $-1,03^{\star \star *}$ & $-1,04^{* * *}$ & $-0,63^{* \star *}$ & $-0,03$ & $-0,91$ \\
\hline Edad2 & $0,01^{* * *}$ & $0,01^{* \star *}$ & $0,01^{*}$ & 0,00 & 0,01 \\
\hline \multicolumn{6}{|l|}{ Submuestra } \\
\hline Lunes-jueves & ref & ref & ref & ref & ref \\
\hline Fin de semana & $5,38^{* * *}$ & $3,73^{* * *}$ & $2,51^{\star \star *}$ & $13,53^{* * *}$ & $8,52^{* * *}$ \\
\hline \multicolumn{6}{|l|}{ Tipo de pareja } \\
\hline Casados & ref & ref & ref & ref & ref \\
\hline Cohabitantes & $2,78^{\star * *}$ & $2,73^{* *}$ & $4,75^{\star \star \star}$ & 2,38 & $-6,98^{*}$ \\
\hline \multicolumn{6}{|l|}{ Educación } \\
\hline Primaria & ref & ref & ref & ref & ref \\
\hline Secundaria & $2,53^{\star * *}$ & $3,09^{* * *}$ & $2,56^{\star \star \star}$ & $5,9^{* * *}$ & 0,14 \\
\hline Universitaria & $6,24^{\star \star *}$ & $6,34^{\star * \star}$ & $6,06^{\star \star \star}$ & $8,88^{\star \star *}$ & $-5,17^{\star *}$ \\
\hline \multicolumn{6}{|l|}{ Actividad laboral } \\
\hline Tiempo completo & ref & ref & ref & ref & ref \\
\hline Tiempo parcial & $11,06^{* * *}$ & $11,28^{* * *}$ & $11,71^{\star \star \star}$ & $11,03^{*}$ & 4,2 \\
\hline Inactividad & $9,23^{\star \star *}$ & $7,36^{\star \star *}$ & $6,62^{* \star *}$ & $17,58^{\star * *}$ & $10,81^{* \star *}$ \\
\hline \multicolumn{6}{|l|}{ Educación pareja } \\
\hline Primaria & ref & ref & ref & ref & ref \\
\hline Secundaria & 0,82 & $-0,55$ & $1,24^{\star \star \star}$ & $-0,54$ & 0,8 \\
\hline Universitaria & $5,25^{\star \star *}$ & $3,41^{* * *}$ & $6,63^{* * *}$ & $2,7^{*}$ & $4,9^{*}$ \\
\hline \multicolumn{6}{|l|}{ Actividad pareja } \\
\hline Tiempo completo & ref & ref & ref & ref & ref \\
\hline Tiempo parcial & $-5,43^{\star * *}$ & $-5,03^{\star * *}$ & $-5,65^{\star \star \star}$ & $-7,7^{\star * *}$ & $-9,55^{\star * *}$ \\
\hline No trabaja & $-9,75^{\star \star *}$ & $-6,49^{* * *}$ & $-10,41^{* * \star}$ & $-11,07^{\star \star *}$ & $-5,27^{* *}$ \\
\hline \multicolumn{6}{|l|}{ Dif. Ingresos } \\
\hline $\mathrm{H}<\mathrm{M}$ & ref & ref & ref & ref & ref \\
\hline Igual & $-4,31^{* * *}$ & $-4,3^{* * *}$ & $-4,81^{* * *}$ & $-3,29$ & 2,57 \\
\hline$H>M$ & $-7,71^{\star * *}$ & $-6,48^{* * *}$ & $-8,22^{* * \star}$ & $-7^{* * *}$ & 0,68 \\
\hline \multicolumn{6}{|l|}{ Hijos } \\
\hline Número hijos & $-0,4^{* * *}$ & $-0,72^{* *}$ & $-1,05^{* * *}$ & $1,31^{* *}$ & $5,56^{* * *}$ \\
\hline Ayuda & $1,88^{* * *}$ & 0,86 & $1,77^{\star \star *}$ & 1,39 & 0,88 \\
\hline Total tareas & $0,02^{* \star *}$ & $0,01^{* * *}$ & $0,02^{* \star *}$ & $0,05^{\star \star *}$ & $0,03^{\star \star \star}$ \\
\hline $\mathrm{N}$ & 9650 & 8576 & 9404 & 6496 & 2728 \\
\hline
\end{tabular}

${ }^{*} p<, 1 ;{ }^{* *} p<, 05 ;{ }^{* * *} p<, 01$ 
Tabla 3.

Resultados del análisis de regresión sobre contribución a las tareas (mujeres)

\begin{tabular}{|c|c|c|c|c|c|}
\hline & Tiempo total tareas & Limpieza & Cocina & Compras & Ocasionales \\
\hline Edad & $1,12^{\star \star *}$ & $1,06^{\star * *}$ & $0,8^{* \star *}$ & 0,35 & 0,65 \\
\hline Edad2 & $-0,01^{* * *}$ & $-0,01^{* * *}$ & $-0,01^{\star * *}$ & $-0,01$ & $-0,01$ \\
\hline \multicolumn{6}{|l|}{ Submuestra } \\
\hline Lunes-jueves & ref & ref & ref & ref & ref \\
\hline Fin de semana & $-5,4^{\star \star *}$ & $-3,77^{* * *}$ & $-2,49^{\star \star *}$ & $-13,53^{* * *}$ & $-8,14^{\star * *}$ \\
\hline \multicolumn{6}{|l|}{ Tipo de pareja } \\
\hline Casados & ref & ref & ref & ref & ref \\
\hline Cohabitantes & $-2,64^{* * *}$ & $-3,14^{* *}$ & $-4,73^{* * *}$ & $-2,26$ & $8,53^{* *}$ \\
\hline \multicolumn{6}{|l|}{ Educación } \\
\hline Primaria & ref & ref & ref & ref & ref \\
\hline Secundaria & $-0,78$ & 0,41 & $-1,23^{* *}$ & 0,56 & $-0,19$ \\
\hline Universitaria & $-5,32^{\star * *}$ & $-3,73^{\star \star *}$ & $-6,73^{\star * *}$ & $-2,61$ & $-4,51$ \\
\hline \multicolumn{6}{|l|}{ Actividad laboral } \\
\hline Tiempo completo & ref & ref & ref & ref & ref \\
\hline Tiempo parcial & $5,54^{\star \star *}$ & $5,12^{\star * *}$ & $5,74^{* \star *}$ & $7,56^{\star \star *}$ & $9,25^{\star *}$ \\
\hline Inactividad & $9,8^{* * *}$ & $6,49^{\star * *}$ & $10,51^{* * *}$ & $11^{* * *}$ & $5,26^{* *}$ \\
\hline \multicolumn{6}{|l|}{ Educación pareja } \\
\hline Primaria & ref & ref & ref & ref & ref \\
\hline Secundaria & $-2,64^{\star \star *}$ & $-3,14^{* * *}$ & $-2,84^{\star * *}$ & $-5,94^{* \star *}$ & $-0,5$ \\
\hline Universitaria & $-6,45^{* * *}$ & $-6,51^{* * *}$ & $-6,31^{* * *}$ & $-8,93^{\star \star \star}$ & 4,38 \\
\hline \multicolumn{6}{|l|}{ Actividad pareja } \\
\hline Tiempo completo & ref & ref & ref & ref & ref \\
\hline Tiempo parcial & $-10,95^{\star \star *}$ & $-10,56^{* * *}$ & $-11,54^{\star * *}$ & $-11,24^{*}$ & $-4,71$ \\
\hline No trabaja & $-9,18^{\star * *}$ & $-7,26^{\star * *}$ & $-6,25^{\star * *}$ & $-17,75^{* * *}$ & $-11,92^{* * *}$ \\
\hline \multicolumn{6}{|l|}{ Dif. Ingresos } \\
\hline $\mathrm{M}<\mathrm{H}$ & ref & ref & ref & ref & ref \\
\hline Igual & $-3,53^{* \star *}$ & $-2,20^{* * *}$ & $-3,56^{\star * *}$ & $-3,53^{* *}$ & $-1,81$ \\
\hline $\mathrm{M}>\mathrm{H}$ & $-7,83^{* * *}$ & $-6,61^{* * *}$ & $-8,29^{* * *}$ & $-6,99^{* \star *}$ & 1,04 \\
\hline \multicolumn{6}{|l|}{ Hijos } \\
\hline Número hijos & $0,46^{*}$ & $0,62^{*}$ & $0,92^{* * *}$ & $-1,32^{\star *}$ & $-4,86^{* * *}$ \\
\hline Ayuda & $-1,86^{* * *}$ & $-0,86^{* * *}$ & $-1,78^{* * *}$ & $-1,28$ & $-0,61$ \\
\hline Total tarea & $-0,02^{* * *}$ & $-0,01^{* * *}$ & $-0,02^{* * *}$ & $-0,05^{\star \star \star}$ & $-0,03$ \\
\hline Constante & $59,95^{\star * *}$ & $65,6^{\star \star *}$ & $66,8^{* * *}$ & $76,99^{\star \star *}$ & $24,49^{*}$ \\
\hline $\mathrm{N}$ & 9662 & 8603 & 9418 & 6512 & 2740 \\
\hline
\end{tabular}


Las diferencias entre estos resultados y los de la tabla 3 , que recoge los modelos para las mujeres, son notables, ya que las tablas son casi simétricas. Comenzando por la primera columna, que representa los coeficientes para la contribución a las tareas totales del hogar, vemos que los factores asociados a una reducción de la proporción de tareas realizadas son: vivir en una pareja no casada, tener un nivel educativo elevado o que lo tenga el otro miembro de la pareja, y el hecho de recibir ayuda con las tareas. Además las mujeres reducen su contribución los fines de semana. Todos estos efectos son contrarios a los que encontrábamos en el caso de los hombres, sin embargo, encontramos efectos del mismo signo en la actividad laboral y en las diferencias de ingresos. Tanto hombres como mujeres reducen la proporción de tareas del hogar que realizan si sus parejas no trabajan o trabajan menos de jornada completa; y aumentan su dedicación cuando

Tabla 4.

Resultados del análisis de regresión sobre el tiempo dedicado a las tareas en parejas del mismo sexo

\begin{tabular}{lccccc}
\hline & Tiempo total tareas & Limpieza & Cocina & Compras & Ocasionales \\
\hline Edad & 7,89 & 5,4 & 2,57 & $-1,4$ & $-1,49$ \\
Edad2 & $-0,07$ & $-0,06$ & $-0,02$ & 0 & 0,02 \\
\hline Submuestra & & & & & \\
\hline Lunes-jueves & ref & ref & ref & ref & ref \\
Fin de semana & $-16,66$ & 2,43 & $-9,54$ & 1,03 & $-10,58$ \\
\hline Educación & & & & & ref \\
\hline Primaria & ref & ref & ref & ref \\
Secundaria & 29,19 & 13,97 & 20,82 & $22,81^{* *}$ & $-28,42^{* *}$ \\
Universitaria & 45,87 & 24,83 & 21,17 & $21,54^{*}$ & $-21,67^{*}$ \\
\hline Actividad & & & & & \\
\hline Trabaja & ref & ref & ref & ref & ref \\
No trabaja & $160,166^{* * *}$ & $85,09^{* * *}$ & $52,77^{* * *}$ & 11,19 & 11,13 \\
M<H & ref & ref & ref & ref & ref \\
Igual & $-69,40 * * *$ & $-28,22$ & $-33,29^{*}$ & $-9,59^{* * *}$ & 1,69 \\
M>H & $-80,67^{* * *}$ & $-26,22$ & $-48,87^{* * *}$ & $-16,34^{* * *}$ & 10,76 \\
\hline Sexo & 23,82 & 17,22 & $-12,45$ & $12,62^{*}$ & 6,43 \\
\hline Constante & $-83,89$ & $-98,57$ & 11,12 & $-48,40$ & 51,97 \\
\hline R2 & 0,44 & 0,31 & 0,38 & 0,19 & 0,19 \\
\hline
\end{tabular}

${ }^{*} p<, 1 ;{ }^{* *} p<, 05 ;{ }^{* * *} p<, 01$

${ }^{*}$ Controlando por el número de niños presentes en el hogar 
son ellos los que no trabajan tantas horas. En el caso de los ingresos, hombres y mujeres reducen su colaboración cuando sus ingresos son más elevados que los de sus parejas y la magnitud de los efectos observados no muestra asimetrías de género. Si examinamos las siguientes columnas de la tabla, los resultados para las actividades más específicas muestran un patrón similar al observado en el total de actividades y que los efectos son más significativos para las actividades rutinarias o femeninas. Formar una unión no casada se asocia en el caso de las mujeres a un aumento del 8,5 por ciento en la proporción de actividades ocasionales realizadas, actividades que incluyen básicamente el bricolaje y cuidados de coche y jardín. El tiempo total que dedica el hogar a las tareas domésticas tiene un efecto significativo sobre la proporción realizada por las mujeres. Al igual que para los hombres, no alcanza el 0,5 por ciento, pero en este caso es negativo.

En el caso de las parejas homosexuales, y quizá debido al reducido número de casos, muy pocas de las variables estudiadas muestran efectos significativos para todos los tipos de actividad. En este caso la variable dependiente que estudiamos es el tiempo total dedicado a las tareas en minutos, por lo que los coeficientes son directamente interpretables como minutos. El efecto más notable es el de la no actividad laboral (por desempleo o inactividad), que aumenta en 160 minutos el tiempo dedicado a las tareas en general, en 85 minutos el dedicado a la limpieza y en 52 el de cocina. La magnitud del efecto apunta a la existencia de especialización en las parejas en las que uno de los miembros no trabaja. Otro efecto muy significativo es el de las diferencias de ingresos: cuando se gana lo mismo o más que la pareja, el tiempo dedicado a las tareas se reduce, 80 minutos en el caso de los que ganan más, y esta reducción es significativa para todas las tareas salvo para las ocasionales. Ambas variables apuntan a la validez de la teoría de los recursos relativos. Finalmente, los individuos con niveles educativos más altos dedican más tiempo a las compras y menos a las tareas ocasionales, tal vez como consecuencia de un poder adquisitivo más alto.

\section{DIscusión}

La primera explicación que hemos considerado en este estudio es la de los recursos relativos. Según estas teorías, deberíamos observar que el miembro de la pareja con mayor potencial en el mercado de trabajo dedica menos tiempo a las labores del hogar. En nuestro caso, podemos medir el potencial en el mercado laboral a través de los ingresos relativos y del nivel educativo, y a través del tipo de actividad podemos evaluar tanto la situación en el mercado de trabajo como la disponibilidad temporal. El gráfico 1 presenta los coeficientes de la diferencia de ingresos en las parejas heterosexuales, y en él podemos apreciar que, tanto la igualdad de ingresos, como tener más ingresos que el otro miembro de la pareja, reducen la proporción de tiempo invertido en las tareas del hogar (tomando como referencia la situación de ganar menos que la pareja). Este efecto tiene la misma dirección tanto para hombres como para mujeres, aunque la magnitud es 
Gráfico 1.

Efecto de la diferencia de ingresos, por sexo

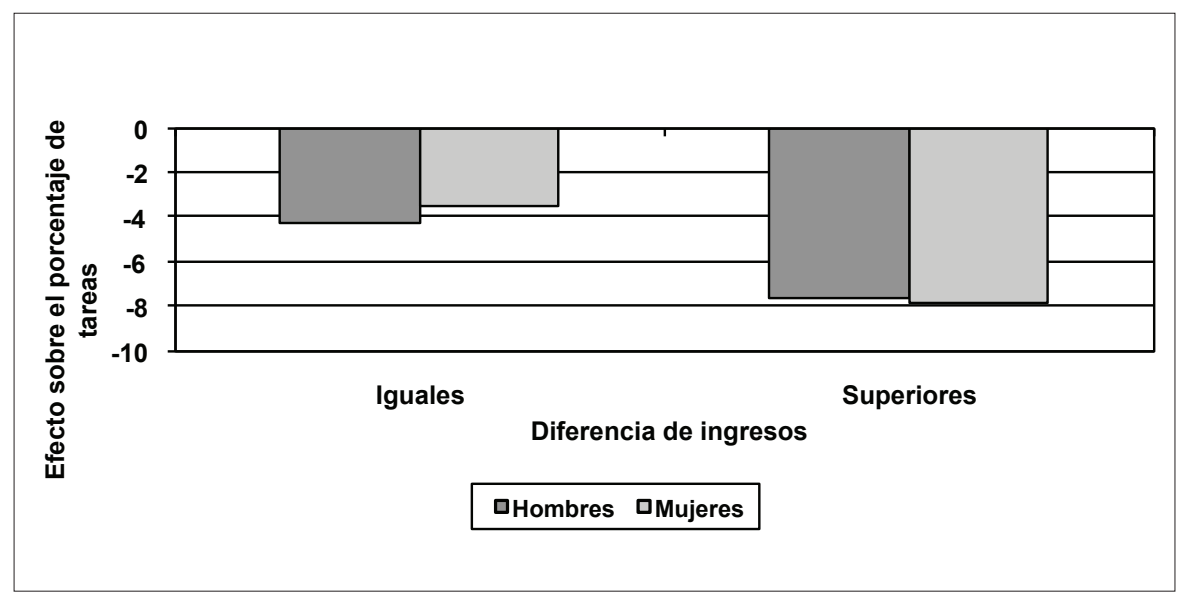

Fuente: Elaboración propia a partir de la EET 2003.

Gráfico 2.

Efecto del tiempo de trabajo, por sexo

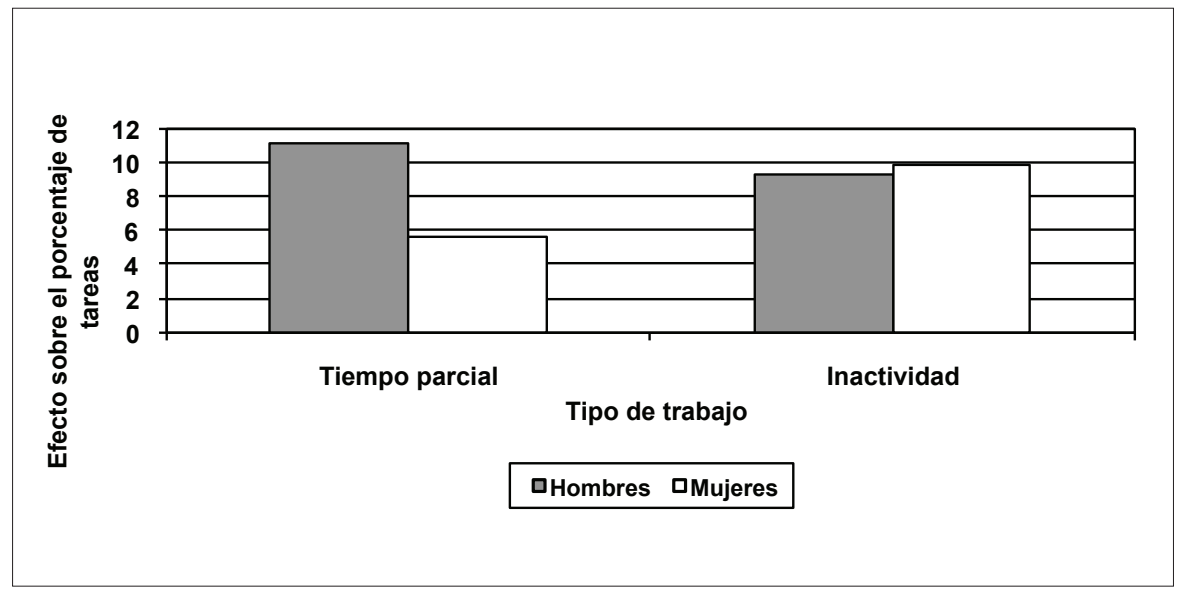

Fuente: Elaboración propia a partir de la EET 2003. 
algo mayor para los hombres, pero esto apuntaría a la validez de la teoría de los recursos relativos. El caso de las parejas del mismo sexo no es directamente comparable porque no mide las diferencias en proporción, pero el resultado apuntaba a un gran incrememto en el tiempo dedicado a las tareas para los que no trabajan, y un importante descenso para los que aportan más ingresos que sus parejas al hogar.

Por otra parte, la hipótesis de la disponibilidad de tiempo mantenía que los trabajadores a tiempo completo se dedicarían menos a las tareas. Si observamos el gráfico 2, vemos, que cuando aumenta la disponibilidad de tiempo y se trabaja a tiempo parcial o no se trabaja, aumenta la proporción de tareas realizadas (en este caso la referencia es trabajar a tiempo completo), de nuevo para ambos sexos; y de nuevo, con un efecto mayor para los hombres, aunque la diferencia entre el tiempo parcial y la inactividad no es proporcional al aumento de tiempo que implican. La razón puede ser que entre los que no trabajan hay una actividad no observada en nuestro análisis: la búsqueda de empleo.

De nuevo, tomando como referencia el trabajo a tiempo completo, cuando el otro miembro de la pareja tiene más tiempo disponible, tanto hombres como mujeres reducen su participación en las tareas; y este efecto es notable para las mujeres cuya pareja trabaja a tiempo parcial, lo cual confirma la hipótesis de la disponibilidad de tiempo. Así pues, tanto los ingresos relativos como la actividad laboral a tiempo completo reducen la contribución a las tareas para los dos sexos, lo que apoya la hipótesis de los recursos relativos. Con todo, seguimos observando que la magnitud de los efectos no es igual para los hombres y para las mujeres.

Una última variable a considerar es el nivel educativo. La educación es una variable problemática en este caso, ya que se puede asociar a un mayor potencial en el mercado

Gráfico 3.

Efecto de la educación, por sexo

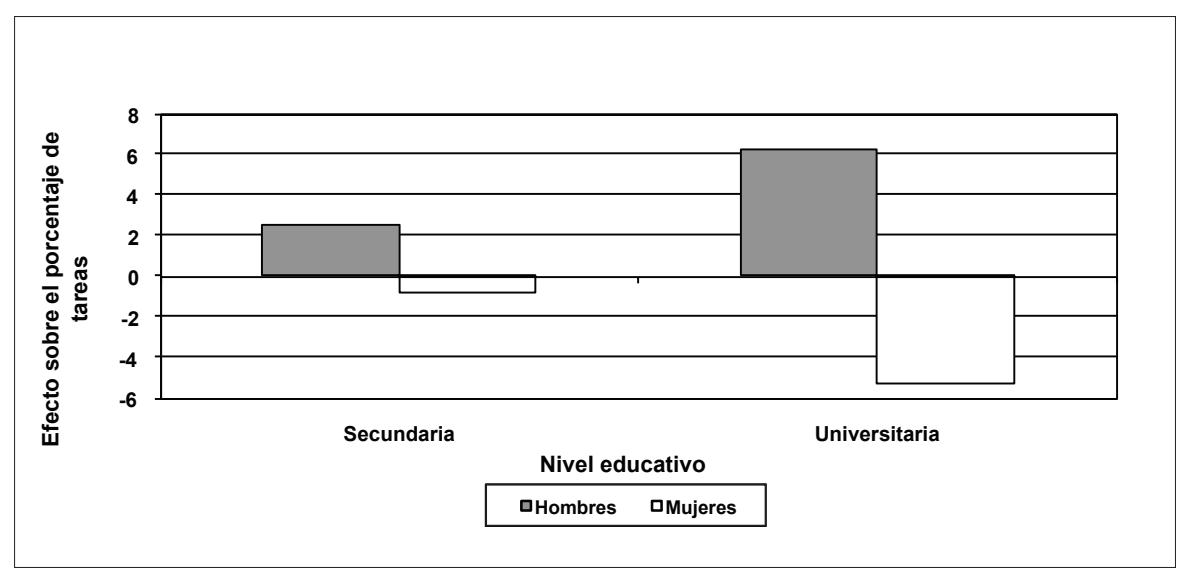

Fuente: Elaboración propia a partir de la EET 2003. 
de trabajo, según la hipótesis de los recursos relativos, pero también a las teorías sobre socialización y género, ya que los niveles educativos más altos se vinculan a valores de género más igualitarios. En el gráfico 3 observamos que el efecto de la educación depende del sexo: tomando como referencia la educación primaria, un nivel educativo más elevado implica mayor participación en las tareas del hogar para los hombres y menor para las mujeres, con un efecto especialmente fuerte en el caso de la educación universitaria. Este efecto se refuerza a través de la educación de la pareja, ya que los hombres que tienen una pareja con nivel educativo alto aumentan también su contribución, mientras que las mujeres con parejas más educadas la reducen, y en la sección anterior hemos visto que el efecto era aún más fuerte para las tareas rutinarias. Este resultado diferenciado por género sugiere que el efecto de la variable no se debe tanto a los recursos relativos como a los valores, ya que si se tratara de recursos, el efecto sería el mismo para ambos sexos, como ocurría en el caso de los ingresos o el tiempo.

Finalmente, en este artículo se proponía introducir el tipo de pareja como variable relevante en los estudios sobre reparto del trabajo doméstico. Al igual que la educación, esta variable no funciona en la misma dirección para los hombres que para las mujeres: en las parejas no casadas las mujeres realizan contribuciones menores al total del hogar, y los hombres mayores, en una magnitud ligeramente superior al 2,5 por ciento. Dado que se estaban controlando otros factores, el efecto de la cohabitación no se debe a diferentes situaciones socioeconómicas o a la juventud de los cohabitantes; más bien parece relacionado con factores no controlados, como los valores, ya que sabemos que los cohabitantes en España tienen ideas más igualitarias en lo que respecta al género (Domínguez y Castro 2008). Esta desigualdad se reproduce en las parejas del mismo sexo: en la sección descriptiva del artículo veíamos que en las parejas formadas por hombres, cada miembro de la pareja realiza una proporción mucho mayor que sus homólogos heterosexuales y en las parejas formadas por mujeres ocurre lo contrario: su contribución es mucho menor que la de las mujeres heterosexuales, siempre dentro de unos tiempos totales distribuidos de forma más igualitaria. No hay que olvidar, sin embargo, que los resultados de este artículo se basan en un número muy reducido de casos, pero en el futuro, si se dispone de fuentes de datos más completas, el estudio de las parejas del mismo sexo puede ayudarnos a discriminar los factores relativos al género y a los recursos, y constituye una prometedora línea de investigación sobre procesos familiares.

Además de su importancia para determinar la validez de la hipótesis de los recursos relativos, los ingresos elevados y la participación en el mercado de trabajo están relacionados con la posibilidad de externalizar servicios y de recibir ayuda para las tareas domésticas. Aquí nos hemos limitado a controlar si se recibe algún tipo de ayuda, pero los resultados no son muy claros ${ }^{4}$. El hecho de recibir ayuda, en principio, podría implicar una división más igualitaria, puesto que la persona que presta la ayuda puede llevar

\footnotetext{
${ }^{4}$ También comprobamos el número de minutos de ayuda recibidos por el hogar, pero los resultados eran similares a los de la variable dicotómica y no se presentan en este artículo.
} 
a cabo las tareas más rutinarias y dejar para el resto del hogar las tareas "preferidas". Pero este efecto depende de las características de la ayuda: en función de si se trata de ayuda desinteresada o pagada, existirá un margen u otro para el tipo de tareas que se realizan; tampoco tendrá el mismo efecto en el reparto que la ayuda se contrate por escasez de tiempo o porque se parta de unos estándares de limpieza muy elevados. Se trata de factores que no podemos controlar con los datos disponibles, pero que podrían mostrar su efecto en futuros estudios.

\section{Conclusiones}

En este artículo hemos analizado la división de las tareas del hogar entre las parejas españolas, utilizando datos de uso del tiempo, que nos proporcionan la estimación más cercana a la realidad de las tareas que se llevan a cabo. Hemos estudiado la proporción de tiempo invertida por cada uno de los miembros de la pareja teniendo en cuenta las teorías existentes sobre la división del trabajo, agrupadas en teorías de la negociación y teorías de la socialización de género. Hemos tenido en cuenta los diferentes tipos de tareas existentes y también el tipo de pareja, examinando las diferencias entre parejas heterosexuales, casadas y no casadas, y parejas del mismo sexo. Se planteaban cuatro hipótesis que intentamos comprobar utilizando datos de la encuesta de Empleo del Tiempo 2003.

Si examinamos las hipótesis planteadas anteriormente en este artículo podemos concluir sobre cada una de ellas que:

(H1) Los recursos relativos influyen en la división del trabajo doméstico. Para todas las tareas consideradas: más recursos implican menos contribución, de una forma neutral respecto al género, con la excepción de la educación.

$(\mathrm{H} 2)$ Algunas de las variables consideradas -el tipo de pareja, día de la semana y nivel educativo- tienen efectos diferentes para hombres y mujeres; para las demás variables, aunque el efecto tenga la misma dirección, no siempre tienen la misma magnitud, con lo que consideramos que estos indicios, a falta de un test directo, apuntan a la validez de las teorías sobre roles de género.

(H3) Encontramos evidencia a favor de la hipótesis: las mujeres hacen una mayor proporción de las tareas en todas las parejas, pero el reparto es más igualitario en proporción de la contribución y en el tipo de tareas realizadas en las parejas no casadas.

$(\mathrm{H} 4)$ Las parejas homosexuales tienen un reparto más igualitario de las tareas del hogar, que parece más influido por los recursos relativos de los miembros de la pareja, pero debemos tener en cuenta el reducido número de casos de nuestro estudio antes de hacer cualquier generalización.

En resumen, hemos encontrado evidencia a favor de las hipótesis de los recursos relativos y la disponibilidad de tiempo, pero también indicios que apuntan a un efecto 
de los roles de género: el hecho de que los efectos tengan magnitudes diferentes para hombres y mujeres, y la existencia de variables con efectos contrarios por sexo, como la educación y el tipo de pareja.

Esta investigación tiene varias limitaciones que hemos señalado a lo largo del artículo, que podrían superarse en el futuro. Una de las principales es que se trata de un estudio transversal. Muchos de los factores que consideramos interesantes a la hora de explicar la división del trabajo, como el estatus laboral, la disponibilidad de tiempo 0 los ingresos cambian a lo largo del tiempo. Observar esos cambios y analizar si tienen un efecto en la dedicación a las tareas es la forma más adecuada de comprobar si existen efectos causales. Además, la organización de los hogares se ve afectada de forma directa por los cambios que se producen en el ciclo vital. El nacimiento de un hijo, por ejemplo, puede cambiar los patrones de uso del tiempo y su distribución entre los miembros de la familia, y esto no podemos observarlo todavía con los datos actuales en el caso español.

Finalmente, dado que la encuesta analizada no incluye información sobre valores ni preferencias, sería importante tenerlos en cuenta en estudios futuros, para ver cómo se adaptan los roles ideales a las situaciones reales, y para dar cuenta de la heterogeneidad de las preferencias individuales, ya que en general asumimos que todos los individuos prefieren un reparto igualitario y parte de la población podría tener una visión distinta.

\section{REFERENCIAS BibLIOGRÁFICAS}

Anxo, D. 2002. "The allocation and the gender division of labour in France and Sweden." Presentado en Symposium France ILO, 2002:99-108.

Balcells, L. 2009. "Analyzing the division of household labor within Spanish families." Revista Internacional de Sociología 67:85-103.

Batalova, J. and P. N. Cohen. 2002. "Premarital Cohabitation and Housework: Couples in CrossNational Perspective." Journal of Marriage and the Family 64:129-144.

Baxter, J. 2005. "To marry or not to marry. Marital status and the household division of labor." Journal of Family Issues 26:300-321.

Becker, G. 1987. Tratado sobre la familia. Madrid: Alianza Universidad.

Bianchi, S. Z. et al. 2000. "Is anyone doing the housework? Trends in the Gender Division of Household Labour." Social Forces 79:191-228.

Black, D. et al. 2000. "Demographics of the Gay and Lesbian Population in the United States: Evidence from Available Systematic Data Sources." Demography 37:139-154. 
Brines, J. 1994. "Economic Dependency, Gender, and the Division of Labor at Home." American Journal of Sociology 100:652-688.

Castro, T. y M. Domínguez. 2008. "Matrimonios sin papeles: perfil sociodemográfico de las parejas de hecho en España según el Censo 2001." Politica y Sociedad 45:47-71.

Clarkberg, M. 1999. "The Price of Partnering: The Role of Economic well-Being in Young Adults' First Union Experiences." Social Forces 77:945-968.

Clarkberg, M., R. Stolzberg and L. White. 1995. "Attitudes, Values and entrance into Cohabitation versus Marital Unions." Social Forces 74:180-192.

Coltrane, S. 2000. "Research on Household Labour: Modelling and Measuring the Social Embededness of Routine Family Work." Journal of Marriage and the Family 62:1208-1033.

Davis, S. N. and T. N. Greenstein. 2004. "Cross-National Variations in the Division of Household Labor." Journal of Marriage and the Family 66:1260-1271.

Domíngez, M. and T. Castro. 2008. "Women's changing socioeconomic position and union formation in Spain and Portugal." Demographic Research 19:1513-1550.

Dribe, M. and M. Stanfors. 2009. "Does Parenthood Strengthen a Traditional Household Division of Labor? Evidence from Sweden." Journal of Marriage and the Family 71:33-45.

Gjerdingen, D. K. and B. A. Center. 2005. "First-Time Parents and Postpartum Changes in Employment, Childcare and Housework Responsabilities." Social Science Research 34:103-116.

Gershuny, J. 2000. Changing Times: Work and Leisure in Postindustrial Society. Oxford: Oxford University Press.

González López, M. J. 2001. "Spouse's Employment Careers in Spain." en Pp. 146-173 Careers of Couples in Contemporary Societies. From Male Breadwinner to Dual Earner Families, edited by H-P. Blossfeld and Sonia Drobnic. Oxford: Oxford University Press.

Gupta, S. 1999. "The effects of transitions in Marital Status on Men's Performance of Housework." Journal of Marriage and the Family 61:700-711.

Hakim, C. 2000. Work-Lifestyle Choices in the 21st century. Nueva York: Oxford University Press.

Hallberg, D. and A. Klevmarken. 2003. "Time for children: a study of parents' time allocation." Journal of Population Economics 16:205-226.

Heuveline, P. and J. Timberlake. 2004. "The Role of Cohabitation in Family Formation: The United States in Comparative Perspective." Journal of Marriage and the Family 66: 1214-1230.

Instituto Nacional de Estadística. 2004. Encuesta de Empleo del tiempo 2002-2003. Tomo I. Metodología y Resultados Nacionales. Madrid: INE.

Kurdek, L. A. 2001. "Differences between heterosexual non-parent couples and gay, lesbian, and heterosexual parent couples." Journal of Family Issues 22:727-754. 
Kurdek, L. A. 2007. "The Allocation of Household Labor by Partners in Gay and Lesbian Couples." Journal of Family Issues 28:132-148.

Lee, Y. and L. Waite. 2005. "Husbands' and wives' Time Spent on Housework: A Comparison of Measures." Journal of Marriage and the Family 67:328-336.

Lennon, M. C. and S. Rosenfield. 1994. "Relative Fairness and the Division of Housework: The Imnportance of Options." American Journal of Sociology 100:506-531.

Lück, D. and D. Hofäcker. 2003. "Rejection and Acceptance of the Male Breadwinner Model. Which Preferences do Women have under which Circumstances?" Globalife Working Papers 60.

Lundberg, S. and R. A. Pollak. 1996. "Bargaining and Distribution in Marriage." Journal of Economic Perspectives 10:139-158.

Meil Landwerlin, G. 1997. "La redefinición de la división del trabajo doméstico en la nueva familia urbana española." Revista Española de Investigaciones Sociológicas 80:69-94.

Meil Landwerlin, G. 2005. "El reparto desigual del trabajo doméstico y sus efectos sobre la estabilidad de los proyectos conyugales." Revista Española de Investigaciones Sociológicas 111:163-180.

Ono, H. 2003. "Women's Economic Standing, Marriage Timing and Cross-National Contexts of Gender." Journal of Marriage and the Family 65:275-286.

Perkins, W. H., and D. de Meris. 1996. "Gender and family effects on the "second shift" domestic activity of college-educated young adults." Gender and Society 10:78-93.

Pittman, J. F. 1995. "The effects of work history and timing of marriage on the division of household labor: a life-course perspective." Journal of Marriage and the Family 58:78-90.

Presser, H. B. 1994. "Employment schedules among dual-earner spouses and the division of household labor by gender." American Sociological Review 59:348-364.

Sayer, L. C., S. M. Bianchi and J. P. Robinson. 2004. "Are parents investing less in children? Trends in mothers' and fathers' time with children." American Journal of Sociology 110:1-43.

Shelton, A. and D. John. 1996. "The Division of Household Labour." Annual Review of Sociology 22:299322.

Solomon, S. E., E. D. Rothblum and K. F. Balsam. 2005. "Money, Housework, Sex, and Conflict: SameSex Couples in Civil unions, Those Not in Civil Unions, and Heterosexual Married Siblings." Sex Roles 52:561-575.

South, S. J. and G. Spitze. 1994. "Housework in Marital and Nonmarital Households." American Sociological Review 59:327-347.

Sullivan, M. 1996. "Rozzie and Harriet? Gender and Family Patterns of Lesbian Coparents." Gender and Society 10:747-767.

Thompson, L., and A. J. Walker. 1989. "Gender in families. Women and men in marriage, work and parenthood." Journal of Marriage and the Family, 51:845-871. 
Twiggs, J. E., J. McQuillan y M. M. Ferrere. 1999. "Meaning and measurement: reconceptualizing measures of household labor." Journal of Marriage and the Family 61:712-724.

Organización de las Naciones Unidas. 2000. Household Accounting: Experiences in Concepts and Computations. Vols. 1 y 2. Nueva York: Naciones Unidas.

West, C. y S. Fenstermaker. 1993. "Power and the accomplishment of gender." Pp. 151-174 en Theory on gender/feminism on theory, edited by P. England. Nueva York: Aldine de Gruyter.

MARTA DOMÍNGUEZ FOLGUERAS es doctora en Sociología por la Universidad Complutense de Madrid y doctora miembro del Instituto Juan March. Su investigación se centra en la sociología de la familia y la demografía. En la actualidad es profesora lectora en el departamento de Ciencias Políticas y Sociales de la Universidad Pompeu Fabra, Barcelona.

RECIBIDO: $26 / 08 / 2009$

ACEPTADO: $16 / 06 / 2010$ 


\section{Anexo}

Distribución de la muestra (\%)

\begin{tabular}{|c|c|c|c|c|c|c|}
\hline & & Mujeres & & & Hombres & \\
\hline & Casadas & Cohabitantes & Mismo sexo & Casados & Cohabitantes & Mismo sexo \\
\hline \multicolumn{7}{|l|}{ Submuestra } \\
\hline Lunes-jueves & 49,63 & 49,03 & 46,10 & 44,94 & 48,54 & 55,77 \\
\hline Fin de semana & 50,37 & 50,96 & 53,90 & 50,06 & 51,46 & 44,23 \\
\hline \multicolumn{7}{|l|}{ Educación } \\
\hline Primaria & 25,86 & 14,29 & 21,29 & 23,42 & 12,02 & 27,36 \\
\hline Secundaria & 54,27 & 51,55 & 55,00 & 53,58 & 59,42 & 48,12 \\
\hline Universitaria & 19,66 & 34,16 & 21,12 & 22,68 & 28,56 & 24,52 \\
\hline \multicolumn{7}{|l|}{ Actividad laboral } \\
\hline Tiempo completo & 37,49 & 61,26 & 38,67 & 79,40 & 85,75 & 79,80 \\
\hline Tiempo parcial & 4,46 & 5,67 & 4,52 & 0,50 & 1,72 & 0,55 \\
\hline Inactividad & 58,05 & 33,07 & 56,81 & 20,11 & 12,53 & 19,66 \\
\hline \multicolumn{7}{|l|}{ Ingresos } \\
\hline$<500$ & 22,16 & 16,70 & 6,28 & 3,13 & 6,06 & 7,58 \\
\hline 500-999 & 40,78 & 48,35 & 40,67 & 29,50 & 39,13 & 35,17 \\
\hline $1000-1249$ & 15,56 & 17,30 & 24,26 & 28,83 & 28,19 & 19,67 \\
\hline $1250-1500$ & 8,32 & 9,82 & 10,58 & 14,95 & 11,43 & 14,82 \\
\hline 1500-1999 & 8,85 & 5,36 & 12,30 & 12,82 & 9,51 & 7,86 \\
\hline$>2000$ & 4,17 & 2,47 & 5,91 & 10,60 & 5,02 & 14,89 \\
\hline Número medio de hijos & 0,72 & 0,49 & 0,60 & 0,72 & 0,49 & 0,60 \\
\hline Edad media & 44,01 & 33,31 & 43,48 & 46,51 & 35,44 & 45,96 \\
\hline $\mathrm{N}$ & 9.074 & 426 & 74 & 9.074 & 426 & 64 \\
\hline
\end{tabular}

